McGill Law Journal

Revue de droit de McGill

\title{
Karounga Diawara, Le contrôle de la puissance de marché : contribution à une approche juridique du marché, Cowansville (Qc), Yvon Blais, 2011, pp 512. ISBN 978-2-89635-516-7
}

\section{Michelle Cumyn}

Volume 57, numéro 3, march 2012

URI : https://id.erudit.org/iderudit/1009070ar

DOI : https://doi.org/10.7202/1009070ar

Aller au sommaire du numéro

Éditeur(s)

McGill Law Journal / Revue de droit de McGill

ISSN

0024-9041 (imprimé)

1920-6356 (numérique)

Découvrir la revue

Citer ce compte rendu

Cumyn, M. (2012). Compte rendu de [Karounga Diawara, Le contrôle de la puissance de marché : contribution à une approche juridique du marché,

Cowansville (Qc), Yvon Blais, 2011, pp 512. ISBN 978-2-89635-516-7]. McGill Law

Journal / Revue de droit de McGill, 57(3), 641-644.

https://doi.org/10.7202/1009070ar d'utilisation que vous pouvez consulter en ligne. 
McGill Law Journal Revue de droit de McGill

\section{RECENSION SIMPLE-}

Karounga Diawara, Le contrôle de la puissance de marché : contribution à une approche juridique du marché, Cowansville (Qc), Yvon Blais, 2011, pp 512. ISBN 978-2-89635-516-7.

L'ouvrage recensé, tiré de la thèse de doctorat de l'auteur, présente un très grand intérêt, même pour les non spécialistes du droit de la concurrence. Il propose une analyse comparée du droit canadien et du droit européen en matière de contrôle des pratiques anticoncurrentielles: ententes, abus de position dominante et fusions. Les deux droits sont clairement présentés, sans simplification excessive, et sans tomber dans les énumérations fastidieuses qui encombrent si souvent les études de droit comparé. La confrontation des solutions retenues par le droit canadien et le droit européen facilite leur analyse critique et conduit l'auteur à suggérer certaines réorientations, tantôt en droit canadien, tantôt en droit européen. L'ouvrage présente un panorama fort intéressant des principales doctrines juridiques et économiques qui animent le droit de la concurrence; en revanche, la dimension politique de ce droit n'est presque pas abordée.

L'ouvrage intéressera les tenants de l'analyse économique du droit ainsi que ceux qui ont critiqué cette approche. L'auteur insiste en effet sur la complémentarité essentielle du droit et de la science économique dans le domaine de la concurrence, et il cherche à préciser le rôle qui revient à chacun. Il prône une approche juridique du marché, qui place l'économie dans un rôle complémentaire par rapport à la règle de droit, lorsqu'il s'agit de mettre en œuvre celle-ci. Le droit doit donc définir ses propres critères à partir des enseignements de la science économique. Ainsi, l'auteur rejette l'analyse économique normative qui, selon lui, entraîne l'instrumentalisation du droit vers un objectif exclusivement économique : la maximisation de l'efficience économique. Il montre également que le modèle hypothéco-déductif employé par la science économique est inapproprié en droit, ce dernier devant intervenir sur le fondement de critères fermes et prévisibles pour les justiciables ${ }^{1}$.

D’un point de vue économique, la puissance de marché désigne la capacité pour une entreprise d'augmenter le prix de ses produits au-dessus

1 Karounga Diawara, Le contrôle de la puissance de marché : contribution à une approche juridique du marché, Cowansville (Qc), Yvon Blais, 2011 aux pp 19-25. 
de leur niveau concurrentiel pendant une certaine période, et de tirer profit de cette augmentation. D'un point de vue juridique, la puissance de marché est associée à l'aptitude qu'a l'entreprise de porter atteinte à la concurrence. Les règles du droit canadien ou européen de la concurrence qui servent à contrôler les ententes, les abus de position dominante et les fusions, lorsque ceux-ci portent atteinte à la concurrence, se fondent sur une démarche en trois étapes, dont chacune fait l'objet d'une partie de l'ouvrage.

Dans une première étape, il convient pour l'autorité de surveillance ou le juge de définir le marché pertinent, c'est-à-dire le lieu où s'exerce la puissance de marché de l'entreprise. Même si la science économique contourne la nécessité de définir le marché pertinent en employant des outils permettant de mesurer directement la puissance de marché, et même si la définition du marché pertinent n'est pas toujours clairement articulée par la loi ou la jurisprudence, il s'agit d'une étape essentielle de l'analyse juridique de la puissance de marché2. En effet, plus le marché est défini de manière large par le juge ou l'autorité de surveillance, moins la position de l'entreprise sur ce marché apparaît comme dominante. Le droit définit le marché en tenant compte à la fois de son étendue géographique et des produits qu'il regroupe et qui sont substituables aux produits de l'entreprise sous examen. Divers indices économiques viennent en aide au droit pour définir le marché pertinent, mais c'est de manière essentiellement autonome qu'il a su dégager ces critères. L'apport essentiel de cette partie de l'ouvrage se trouve dans deux principes dégagés par l'auteur: d'une part, le principe de liaison, en vertu duquel la délimitation du marché pertinent a pour rôle essentiel de circonscrire le pouvoir de marché ; d'autre part, le principe de concrétisation, selon lequel la définition du marché pertinent, bien qu'unitaire, tient compte de l'origine de la puissance de marché et s'adapte ainsi aux exigences formelles et matérielles différentes des règles relatives à l'entente, à l'abus de position dominante et à la fusion.

Dans une seconde étape, l'application des règles relatives aux pratiques anticoncurrentielles suppose une évaluation juridique de la puissance de marché de l'entreprise sous examen, c'est-à-dire de l'étendue de la domination qu'elle exerce sur son marché. De nouveau, la science économique éclaire le droit qui détermine, à l'aide des indices économiques pertinents, quel est le degré de domination exercé par l'entreprise ${ }^{3}$. Le droit européen accorde une plus grande importance que le droit canadien à la part de marché de l'entreprise. L'approche canadienne est jugée pré-

$2 \quad$ Ibid aux pp 37-42.

3 Ibid aux pp 159-80. 
férable par l'auteur, parce que l'analyse économique démontre que la part de marché est un révélateur imparfait de la puissance de marché. Il convient de tenir compte, outre la part de marché, des barrières à l'entrée, dont l'auteur dresse la typologie et pour lesquelles il propose un cadre d'analyse juridique approprié. L'auteur aborde par ailleurs la domination collective du marché, qui s'opère lorsque plusieurs entreprises coordonnent de manière implicite ou explicite leurs actions de manière à maintenir des prix élevés ou à freiner l'innovation ou le développement de nouveaux produits sur ce marché.

Dans une troisième étape, lorsqu'il s'agit d'évaluer la validité des actes entrepris ou projetés par l'entreprise au regard des règles du droit de la concurrence, le droit examine leurs effets anticoncurrentiels. Or, ces effets anticoncurrentiels sont légitimés par le droit lorsque les actes projetés comportent par ailleurs certains effets bénéfiques, par exemple, quand ils permettent la réalisation d'économies d'échelle, le développement de nouveaux produits ou une amélioration de leur qualité : ce sont les " gains en efficience ». L'auteur argue que l'intérêt des consommateurs constitue en définitive le guide le plus sûr, s'agissant de déterminer s'il y a lieu de valider les actes entrepris ou projetés en raison de leurs effets globalement bénéfiques. L'analyse doit donc avoir pour but de déterminer quel est l'effet prévisible pour les consommateurs des actes sous examen. La notion de consommateur revêt ici un sens générique qui englobe " tout individu qui répond à une offre sur le marché indifféremment de son statut socioéconomique ${ }^{4}$. Si les effets globaux de l'exercice de la puissance de marché sont positifs, l'entreprise devrait pouvoir aller de l'avant. Dans le cas contraire, il faut la contrôler.

Peut-être en raison du partage des compétences législatives découlant du cadre constitutionnel canadien, peu de chercheurs au Québec et au Canada mettent en relation le droit de la consommation et le droit de la concurrence, alors que ces matières entretiennent en Europe des liens beaucoup plus étroits. La perspective ouverte par l'auteur, voulant que la protection des consommateurs constitue l'un des piliers du droit de la concurrence, est certes intéressante et mérite réflexion. L'auteur n'a toutefois pas démontré à mon entière satisfaction qu'il faut écarter d'autres objectifs qui sont admis par le droit de la concurrence et qui paraissent, eux aussi, importants, surtout celui de favoriser le développement des PME et de prévenir la trop grande concentration du pouvoir économique. Comme le mentionne l'auteur dans l'introduction de son ouvrage ${ }^{5}$, le contrôle des pratiques anticoncurrentielles relève, en quelque sorte, d'une prérogative

$4 \quad$ Ibid à la p 267, n 783.

$5 \quad$ Ibid aux pp 2-3. 
644 (2012) 57:3 MCGiLl LAW JournAL REVUe DE DroIt DE MCGILL

régalienne de l'État; or les États ne devraient-ils pas se préoccuper de la concentration du pouvoir économique qui représente une menace à leur souveraineté ? Si le droit de la concurrence s'oriente vers la satisfaction des consommateurs, la dimension politique de ce droit ne risque-t-elle pas d'être occultée?

Michelle Cumyn 